21st Particles and Nuclei International Conference (PANIC 2017)

International Journal of Modern Physics: Conference Series

Vol. 46 (2018) 1860007 (9 pages)

(C) The Author(s)

DOI: $10.1142 / \mathrm{S} 2010194518600078$

\title{
Development of Detectors for Physics at the Terascale
}

\author{
Attilio Andreazza \\ Dipartimento di Fisica, Università degli Studi di Milano, and INFN, \\ Sezione di Milano, Via Celoria 16, Milano, I-20133, Italy \\ attilio.andreazza@mi.infn.it
}

Published 3 May 2018

\begin{abstract}
The detector systems for particle physics experiment at the future high-energy and highluminosity colliders will need to improve resolution, radiation hardness, and rate capability with respect to the current generation of experiments. Many promising technological solutions are being developed for both tracking detectors and calorimeters.
\end{abstract}

Keywords: Particle Detectors; Colliders; Tracking Detectors, Calorimetry.

\section{Setting the scene}

Future high-energy-physics colliders push the requirements for the experimental apparatuses beyond the current state-of-art. The technological challenges, of course, depend on the physics case. This contribution focuses on the next generation of accelerators at the high-energy frontier, considering mainly detector under construction or design to operate during the Run 3 (2021-2023) and the high luminosity run (2026-2035) of the LHC or at possible $e^{+} e^{-}$colliders.

At high-energy hadron-colliders, the total inelastic $p-p$ cross section is 100 $120 \mathrm{mb}$ in the 14-100 TeV center-of-mass energy range, resulting high-rate and highmultiplicity interactions. As a consequence, detectors must satisfy harsh radiation hardness requirement and must develop solution to mitigate the effect of multiple $p-p$ interactions in the same bunch crossing (pileup). At the High Luminosity LHC (HL-LHC) upgrade, $^{1}$ with a design luminosity of $7 \times 10^{34} \mathrm{~cm}^{-2} \mathrm{~s}^{-1}$, he innermost tracking system will sustain a total radiation damage corresponding to a fluence of the order of $10^{16} 1 \mathrm{Mev} n / \mathrm{cm}^{2}$ and detectors will need to cope with a pileup of 200 interactions at $25 \mathrm{~ns}$ bunch crossing spacing. These limits need to be pushed further for future machines at higher energy and luminosities.

This is an Open Access article published by World Scientific Publishing Company. It is distributed under the terms of the Creative Commons Attribution 4.0 (CC-BY) License. Further distribution of this work is permitted, provided the original work is properly cited. 

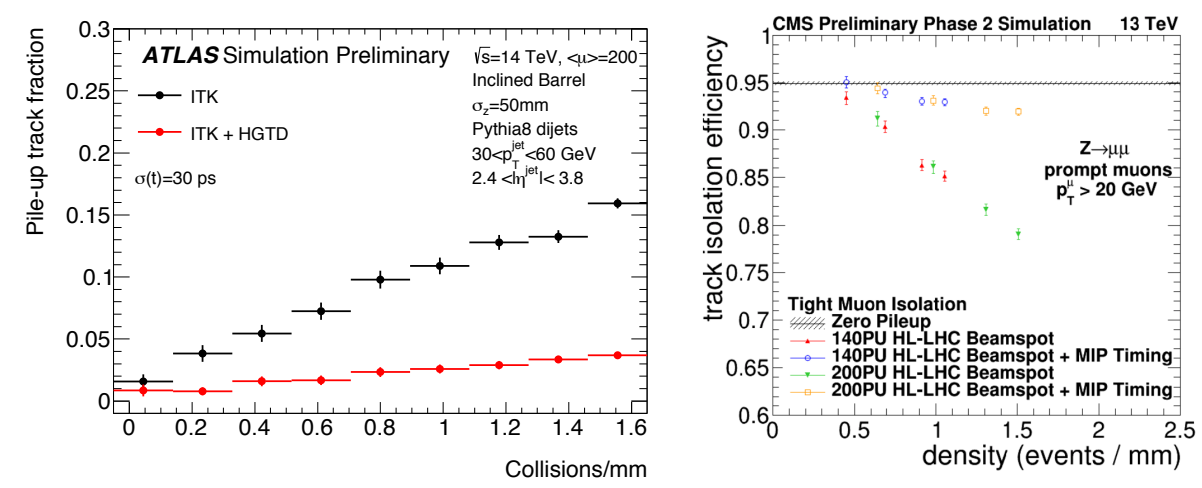

Fig. 1. Examples of performance sensitivity to pile-up at the HL-LHC with and without precision timing information: (left) contamination of a jet from pileup particles ${ }^{2}$ and (right) efficiency of muon isolation criteria. ${ }^{3}$

An important effect of this pileup level, is that with the LHC bunch length $\sigma_{z}=5 \mathrm{~cm}$, the density of interactions make spacial separation between the multiple $p-p$ interaction vertices difficult, especially in the forward detector region. This is illustrated in Fig. 1, where the contamination of charged particles from pileup increases with the density of interactions and it affects also the selection of isolated leptons. Nevertheless, interaction in the same spacial position may actually happen at separate times with $\sigma_{t}=\sigma_{z} / c=180 \mathrm{ps}$, and particles from different interactions can be discriminated with high-resolution time-measurements. This motivates the experiments to deply detectors with a time resolution of $\approx 30 \mathrm{ps}$, as will be shown in Sections 2 and 4.

Plans for future $e^{+} e^{-}$colliders cover center-of-mass energies from the $Z$ mass to above the $t \bar{t}$ thresholds, with luminosities in the same $10^{34}-10^{35} \mathrm{~cm}^{-2} \mathrm{~s}^{-1}$ range. The much lower electroweak cross-section results in more comfortable particle rate and dose and it allows to emphasize high-resolution rather than high-rate and radiation hardness capabilities. Typical requirements are to minimize the multiple scattering term, in order to reach an impact parameter resolution $\sigma_{d} \approx 5 \mu \mathrm{m} \oplus 10-$ $15 \mu \mathrm{m} \cdot \mathrm{GeV} / p$, to design the tracking system for $\sigma_{1 / p}=2-5 \times 10^{-5} \mathrm{GeV}^{-1}$, and aiming to jet energy resolution of 3-4\% at $40 \mathrm{GeV}$, for the identification of $W$ and $Z$ hadronic decays. These goals share with the $p-p$ scenario the direction towards high granularity detectors.

In the following, the solutions that are being developed for the high precision vertex tracking, large volume tracking and calorimetry will be discussed. At first emphasizing the technologies foreseen for the upgrades of the LHC experiments in view of the HL-LHC, followed by some of the most promising approaches for $e^{+} e^{-}$colliders. This is a very personal selection, and, because of time limitations, discussion of trigger, online and offline computing, and particle identification will be omitted, despite their paramount importance in modern high-energy-physics experiments. 

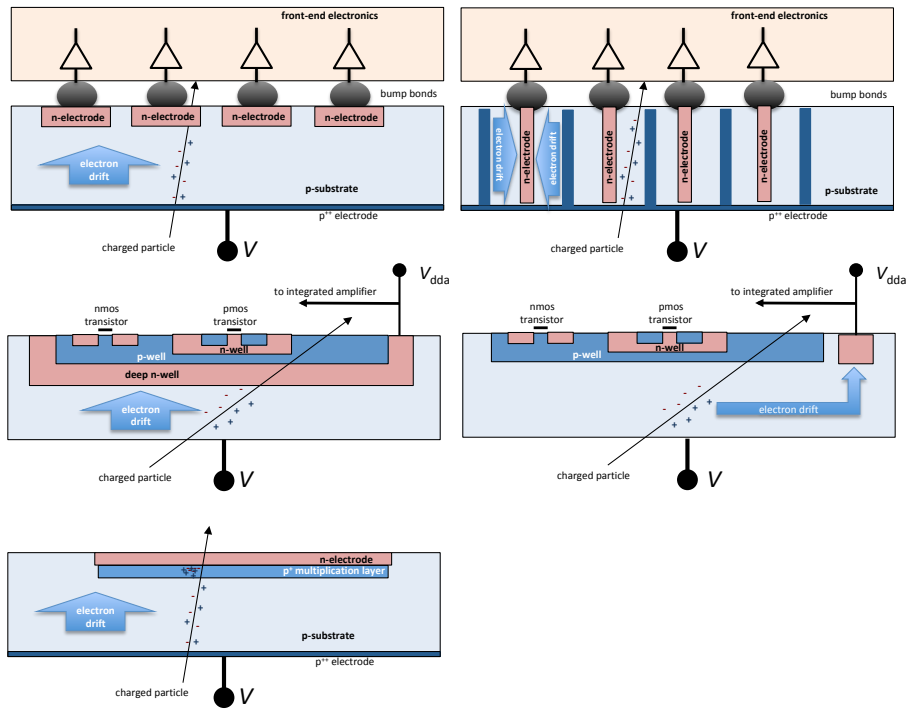

Fig. 2. Different silicon detector technologies considered for the HL-LHC upgrades: (top-left) hybrid planar sensors, (top-right) hybrid 3D sensors, (middle-left) depleted MAPS with large electrode layout, (middle-right) depleted MAPS with small electrode layout, (bottom) LGAD.

\section{Silicon Trackers}

The high particle density and radiation level at the HL-LHC results in the choice of full silicon trackers. ${ }^{4,5}$ With respect to the present detectors, a feature of both tracker upgrades is the increase in coverage of the pixel section, providing tracking up to pseudorapidity $|\eta|<4$.

In order to cope with high doses and rates in the pixel layers different detector configurations are explored. The more relevant are sketched in Fig. 2 and discussed below. The most consolidated approach is using hybrid sensors with separate electronics and sensor dies, connected by bump-bonding. A granularity of $50 \times 50$ or $25 \times 100 \mu \mathrm{m}^{2}$ can be achieved by large scale integration processes. Thin $(100-150 \mu \mathrm{m})$ planar sensors provide lower dark current (and therefore lower noise) and better charge collection efficiency than the thicker devices now in use. The reduced thickness corresponds to a smaller input signal to the front-end electronics. This seems manageable with the first prototypes developed by the CERN RD53 Collaboration in the $65 \mathrm{~nm}$ TSMC technology. ${ }^{6}$ Higher radiation hardness for the innermost pixel layer can be achieved by $3 \mathrm{D}$ sensors, where the lateral depletion requires a significantly smaller bias voltage to achieve the complete depletion and a high electric field for fast charge collection. Ref. 7 provides an overview of the performance of recent prototypes.

Besides the high-rate/high-radiation frontier, heavy-ions and lepton collider experiments aim to improve performance at the high-resolution frontier. In these 


\section{A. Andreazza}

environments the critical issue is to combine fine granularity with low mass. It is obtained by using thin detectors with low power consumption, to minimize the material of the cables and cooling system. This is the territory of detectors integrating in the same die both the active volume and the first amplifier stage. Some detector currently under construction are the DEPFETs of BELLE II pixel detector, ${ }^{8}$ with a material thickness of only $0.1 \%$ of radiation length per layer, and the monolithic ALPIDE chip of the ALICE experiment, which has a $27 \times 29 \mu \mathrm{m}^{2}$ pixel size and a power consumption $<40 \mathrm{~mW} / \mathrm{cm}^{2},{ }^{9}$ about two orders of magnitude less than the requirement for RD53 readout chip.

Low power monolithic active pixel sensors (MAPS) collect signal by diffusion and therefore have limited time resolution, which make them unsuitable for operation in a high-rate environment. An active line of development, in view of application at the HL-LHC, is the realization of monolithic devices with a depleted substrate where charge is collected by drift, ${ }^{10}$ as displayed in Fig. 2(middle). These devices have better radiation hardness and time performance than standard MAPS. They can implement different design concepts, either with the front-end electronics integrated inside large collection electrodes, providing uniform charge collection at the cost of a moderate input capacitance, or with the front-end electronics integrated outside small collection electrodes, providing low input capacitance at the price of some nonuniformity in charge collection. ${ }^{11,12}$ Depleted substrates can range from relatively thin epitaxial layer to a full high-resistivity wafer bulk with buried layers or SOI. ${ }^{13}$

Finally, a detector that is gaining a lot of interest for timing application is the low-gain avalanche diode (LGAD). ${ }^{14}$ It achieves excellent time resolution by reducing the signal risetime thanks to charge amplification in a high-field region below the collecting electrode, as outlined in Fig. 2(bottom). A time resolution at the level of the $30 \mathrm{ps}$ required for mitigating the effect of pileup at HL-LHC has been reached: Fig. 3(left) shows the observed performance on prototypes realized for the ATLAS High Granularity Timing Detector (HGTD). ${ }^{2}$ The technological challenge is to maintain this time resolution on large systems and preserve it after radiation damage, which tends to degrade the effectiveness of the multiplication layer.

\section{Gas Trackers}

For large volume tracking detectors, like muon systems or the central tracking of $e^{+} e^{-}$colliders, gaseous detectors are the only affordable option. Traditional solutions, like drift chambers and TPCs are the backbone of many experiments under construction or design (MEG, Belle II, ILD to cite a few). Innovation is driven by the maturity reached by micro-pattern gas detectors (MPGD), whose operating principle is sketched in Fig. 4. They rely on the separation between the drift and multiplication volumes provided by micromega meshes $(\mathrm{MM})^{15}$ and gas electron multipliers (GEM). ${ }^{16}$ This results in high rate capabilities and flexibility in the collection electrode design. 

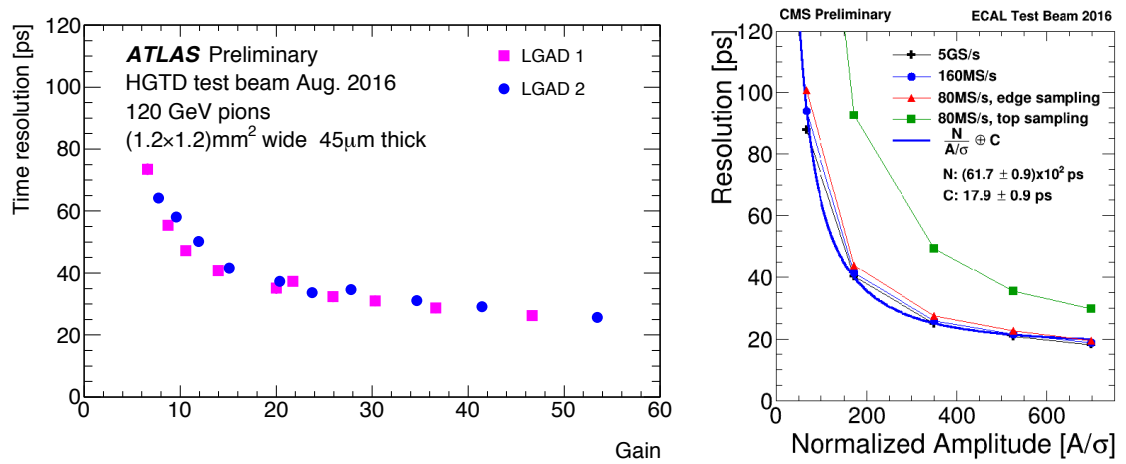

Fig. 3. Test beam time resolution measurement for different sensors: (left) resolution vs. gain for LGADs prototypes of the ATLAS HGTD, ${ }^{2}$ (right) CMS barrel electromagnetic calorimeter resolution vs. normalized pulse amplitude, for different sampling configurations. ${ }^{3}$
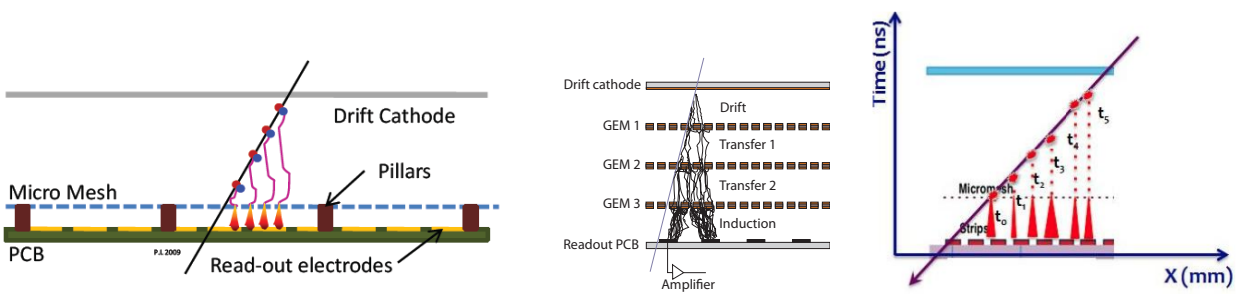

Fig. 4. Sketch of the working principles of some MPGD examples: (left) micromegas, ${ }^{17}$ (middle) triple GEM arrangement, ${ }^{18}$ and (right) the $\mu \mathrm{TPC}^{22}$

In the general purpose LHC experiments, ATLAS and CMS, the first layers of the muon systems at the HL-LHC will sustain a hit rate of $10-15 \mathrm{kHz} / \mathrm{cm}^{2}$ in the forward region and must achieve an angular resolution of $\approx 1 \mathrm{mrad}$ at the trigger level. ${ }^{17,18}$ To achieve such a performance, the ATLAS experiment is rebuilding its Small Wheels in front of the forward toroid with eight layers of thin-gap chambers for trigger and of MM for precision track reconstruction, ${ }^{17}$ and the CMS experiment is planning to instrument the two first forward layers with triple GEM detectors. ${ }^{18}$

For large tracking systems, the new generation of time projection chambers (TPC) uses MPGDs as end-plate electrode instead of multi-wire proportional chambers. The latter have a rate limit of few $\mathrm{kHz}$, due to the dead time introduced by the gating needed to prevent ion backflow in the drift region. This prevents the exploitation of the full luminosity of the LHC and future $e^{+} e^{-}$colliders, that results in the overlapping of multiple events in the drift region. The MPGDs provide an intrinsic limitation of the ion backflow, allowing for continuous operation without gating. This technique, developed for experiments at a linear collider, ${ }^{19}$ now finds application in the ALICE upgrade ${ }^{20}$ for the Run 3 of the LHC, where a quadruple GEM configuration achieves a backflow $<1 \%$. This makes the experiment able to fully profit from the achievable interaction rate. 


\section{A. Andreazza}

A further illustration of the great flexibility of MPGDs is the production of cylindrical GEMs ${ }^{21}$ which can act at the same time as both internal support structure and first measured point of large drift chambers. In such a geometry, the coupling to readout electronics with time resolution at the ns level can be used to measure not only the charged particle crossing point, but also its direction, using the so-called $\mu \mathrm{TPC}$ mode outlined in Fig. 4(right). ${ }^{22}$

\section{Calorimetry}

The clear trend in calorimetry is the increase in detector granularity, both spaceand time-wise. This common direction is motivated by different aspects in different experimental environment.

Calorimeter are a structural asset of particle physics experiments, therefore cannot be easily replaced with new detectors. Nevertheless a significant performance improvement can be be achieved by an upgrade of the readout system. A good example is the $H \rightarrow \gamma \gamma$ detection by the $\mathrm{PbWO}_{4}$ electromagnetic calorimeter of the CMS experiment. To compute the two photons invariant mass, the $H$ production vertex must be known, but the high pileup level at HL-LHC may introduce ambiguities in vertex reconstruction. As said in Sect. 1, hit timing information with 30 ps resolution can identify the decay vertex with sufficient accuracy. This resolution can be achieved, as demonstrated in test beams and shown in Fig. $3,{ }^{3}$ by the combination of cooling, to reduce the noise of irradiated APD, shorter shaping time in the front-end electronics, and increasing the sampling frequency to $160 \mathrm{MHz}$. A similar approach is also followed by the ATLAS experiment, that plans an upgrade of the front-end electronics, making the full detector granularity available at the trigger level. ${ }^{23}$

For high precision detectors at $e^{+} e^{-}$experiments, the goal is to reach a jet energy resolution of $3-4 \%$ at $50 \mathrm{GeV}$, in order to discriminate between $W \rightarrow q \bar{q}$ and $Z \rightarrow q \bar{q}$ decays. This can be achieved by disentangling the electromagnetic and hadronic components of jets.

One approach is the use of particle-flow algorithms, ${ }^{24}$ exploiting a high granularity calorimeter to separate each primary particle contribution to the jet energy, as displayed in Fig. 5(left). Since $\approx 60 \%$ of energy is carried by charged particles and $\approx 30 \%$ by photons, their energy can be taken by the high precision tracking system and electromagnetic calorimeter, relying on the hadronic calorimetry only for the remaining $10 \%$ of energy carried by neutral hadrons. High granularity detectors have been investigated for several years by the CALICE Collaboration, which systematically explored a wide range of options for passive material and readout techniques. ${ }^{25}$ This R\&D program is now finding application in the upgrade of the LHC experiments. Tungsten absorber interleaved with silicon pads of $0.5-1 \mathrm{~cm}^{2}$ size constitute the main component of the ALICE FoCAL,${ }^{26}$ foreseen for the Run 3 of the LHC. It pushes further the high-granularity concept by the addition of few layers of MAPS, providing two-particle separation capabilities at the mm level. 

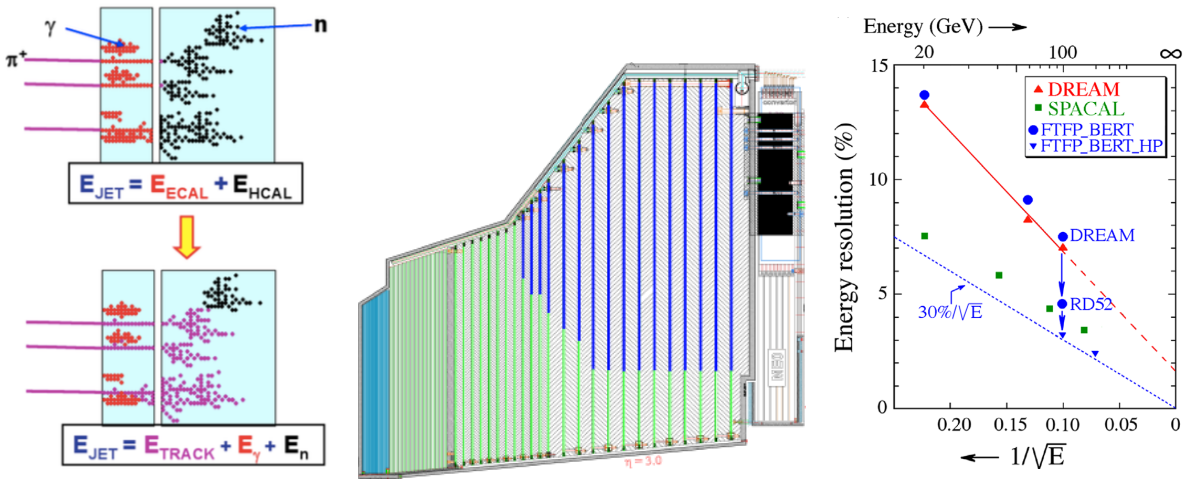

Fig. 5. Different techniques for precision calorimetry: (left) schematics view of the particle flow concept ${ }^{24}$ (middle) layout of the CMS HGCal: ${ }^{27}$ in green are the silicon pads, covering the highdensity/high-dose region, in blue the steel-scintillator hadronic section, and (right) test beam resolution of the RD52 dual-readout calorimeter, compared with simulation and extrapolated to complete solid angle coverage. ${ }^{28}$

The same Si-W approach is implemented in the electromagnetic section of the CMS High-Granularity Calorimeter (HGCal), ${ }^{27}$ illustrated in Fig. 5(middle), that will replace the forward section of CMS electromagnetic calorimeter for the HL-LHC. Silicon pads provide the high granularity and radiation hardness needed also in the part of the hadronic section nearest to the beamline. In the outer part of the hadronic section, high transversal and longitudinal granularity is maintained by the use of steel-scintillator tiles with $3 \times 3 \mathrm{~cm}^{2}$ area. Processing the amount of information due to such a high-granularity is an heavy computational task and the enabling technology is the availability of high-performance FPGA, for fast on-line clustering in order to provide a level-1 trigger decision within the required $5 \mu \mathrm{s}$ latency.

A complementary technique, investigated by the CERN RD52-DREAM Collaboration, is dual readout calorimetry, sampling both scintillation light, sensitive to the $\mathrm{d} E / \mathrm{d} x$ of charged particles, and Cherenkov light, produced mainly by $e^{ \pm}$and therefore proportional to the shower electromagnetic component. Simulation, confirmed by the test beam data illustrated in Fig. 5(right), shows that the energy resolution goal can be reached. ${ }^{28}$ This technique is proposed for future $e^{+} e^{-}$colliders and developments are ongoing, aiming to increase the segmentation by using compact SiPM arrays.

\section{Conclusions}

Innovations in detector techniques are continuing to improve the performance of particle physics apparatuses. 


\section{A. Andreazza}

The upgrades of the LHC experiments for Run 3 and the HL-LHC show that silicon detectors are still the technology of choice for high precision tracking, while new MPGDs provide the resolution and high-rate capability for muon systems.

It is possible to identify one common direction which is the increase of granularity, both in space and time: finely segmented calorimeters are being deployed both at hadron and lepton colliders, while timing with 30 ps precision is emerging as a resource against the high pileup of hadron colliders.

\section{References}

1. G. Apollinari et al. (eds.), High-Luminosity Large Hadron Collider (HL-LHC): Technical Design Report V. 0.1 CERN-2017-007-M (CERN, Geneva, 2017).

2. D. Boumediene, PoS(EPS-HEP2017)485 (2017); B. Lenzi, A High-Granularity Timing Detector for the Phase-II upgrade of the ATLAS Calorimeter system, http://indico.ihep.ac.cn/event/6387/session/16/contribution/88 (accessed 24 January 2018).

3. N. Marinelli, PoS(EPS-HEP2017)497 (2017); P. Meridiani, Precision timing calorimetry with the upgraded CMS ECAL, these proceedings.

4. ATLAS Collaboration, ATLAS Inner Tracker Strip Detector Technical Design Report, ATL-TDR-025 CERN-LHCC-2017-005 (CERN, Geneva, 2017).

5. CMS Collaboration, The Phase-2 Upgrade of the CMS Tracker, CMS-TDR-014 CERN-LHCC-2017-009 (CERN, Geneva, 2017).

6. RD53 Collaboration (N. Demaria et al.), JIST 11 C12058 (2016).

7. M. Garcia-Sciveres and N. Wermes, Advances in pixel detectors for experiments with high rate and radiation, arXiv:1705.10150 (2017).

8. B. Schwenker, PoS (Vertex 2016) 011.

9. S. Beolé, PoS (Vertex 2016) 014.

10. I. Peric, Nucl. Instr. and Methods A $\mathbf{5 8 2} 876$ (2007).

11. N. Wermes, Nucl. Instr. and Methods A $\mathbf{8 2 4} 48$ (2016).

12. H. Pernegger et al., JINST 12 P06008 (2017).

13. Y. Arai, PoS (Vertex 2016) 029.

14. N. Cartiglia et al., Nucl. Instr. and Methods A 84547 (2017); H. Sadrozinsky et al., Nucl. Instr. and Methods A 83118 (2016).

15. Y. Giomataris, P. Rebourgeard, J. Robert, and G. Charpak, Nucl. Instrum. and Methods A 37629 (1996).

16. F. Sauli, Nucl. Instrum. and Methods A 386531 (1997).

17. ATLAS Collaboration, New Small Wheel Technical Design Report, ATLAS-TDR-20 CERN-LHCC-2013-006 (CERN, Geneva, 2013).

18. CMS Collaboration, CMS technical design report for the muon endcap GEM upgrade, CMS-TDR-013 CERN-LHCC-2015-012 (CERN, Geneva, 2015).

19. ILC Collaboration, ILC Technical Design Report - Volume 4: Detectors, arXiv:1306.6329 (2013).

20. ALICE Collaboration, Addendum to the Technical Design Report for the Upgrade of the ALICE Time Projection Chamber, ALICE-TDR-016-ADD-1 CERN-LHCC-2015002 (CERN, Geneva, 2015).

21. L. Lavezzi et al., JINST 12 C07038 (2017); A. Balla et al., JINST 9 C01014 (2014).

22. M. iodice, JINST 9 C01017 (2014).

23. ATLAS Collaboration, ATLAS Phase-II Upgrade Scoping Document, CERN-LHCC2015-020 (CERN, Geneva, 2015). 
24. J. Marshall and M. Thomson, Pandora Particle Flow Algorithm, in Proc. of Calorimetry for the High Energy Frontier eds. J.-C. Brient, R. Salerno adn Y. Sirois (École Polytechnique, Paris, 2013) p. 305.

25. G. Grenier, PoS(EPS-HEP2017) 497 (2017).

26. C. Zhang], Nucl. Instrum. and Methods A 845542 (2017).

27. A.-M. Magnan, JINST 12 C01042 (2017); S. Jain, these proceedings.

28. R. Wigmans, Nucl. Instrum. and Methods A 824721 (2016). 